\title{
Demonstration of a Linear Prolongation CMFD Method on MOC
}

\author{
Jin Li, ${ }^{1}$ Yunlin Xu, ${ }^{2}$ Dean Wang, ${ }^{3}$ Qicang Shen, ${ }^{1}$ \\ Brendan Kochunas, ${ }^{1}$ Thomas Downar $^{1}$ \\ ${ }^{1}$ Department of Nuclear Engineering and Radiological Sciences, University of Michigan \\ 2355 Bonisteel Blvd., Ann Arbor, MI 48109 \\ ${ }^{2}$ School of Nuclear Engineering, Purdue University \\ 400 Central Dr., West Lafayette, IN 47906 \\ ${ }^{3}$ Department of Mechanical and Aerospace Engineering, The Ohio State University \\ 201 W 19th Ave., Columbus, OH 43210 \\ lijinthu@umich.edu, yunlin@purdue.edu,wang.12239@osu.edu, \\ qicangsh@umich.edu,bkochuna@umich.edu,downar@umich.edu
}

\begin{abstract}
Coarse Mesh Finite Difference (CMFD) method is a very effective method to accelerate the iterations for neutron transport calculation. But it can degrade and even fail when the optical thickness of the mesh becomes large. Therefore several methods, including partial current-based CMFD (pCMFD) and optimally diffusive CMFD (odCMFD), have been proposed to stabilize the conventional CMFD method. Recently, a category of "higherorder" prolongation CMFD (hpCMFD) methods was proposed to use both the local and neighboring coarse mesh fluxes to update the fine cell flux, which can solve the fine cell scalar flux discontinuity problem between the fine cells at the bounary of the coarse mesh. One of the hpCMFD methods, refered as lpCMFD, was proposed to use a linear prolongation to update the fine cell scalar fluxes.

Method of Characteristics (MOC) is a very popular method to solve neutron transport equations. In this paper, lpCMFD is applied on the MOC code MPACT for a variety of fine meshes. A track-based centroids calculation method is introduced to find the centroids coordinates for random shapes of fine cells. And the numerical results of a 2D C5G7 problem are provided to demonstrate the stability and efficiency of lpCMFD method on MOC. It shows that lpCMFD can stabilize the CMFD iterations in MOC method effectively and lpCMFD method performs better than odCMFD on reducing the outer MOC iterations.
\end{abstract}

KEYWORDS: 1pCMFD, CMFD, MOC, stability, acceleration

\section{INTRODUCTION}

Coarse Mesh Finite Difference(CMFD) method is a very effective method to accelerate the iterations for neutron transport calculation[1]. But it can degrade and even fail when the optical 
thickness of the mesh becomes large. Therefore several methods, including partial current-based CMFD (pCMFD)[2], optimally diffusive CMFD (odCMFD)[3] and linear prolongation CMFD (lpCMFD)[4][5], has been proposed to stabilize the conventional CMFD method.

The pCMFD was developed by Cho and was adopted in nTracer[6]. A linearized Fourier analysis developed by Jarret showed that the pCMFD is theoretically equivalent to a conventional CMFD method when an additional term $\Delta / 4$ is added to the diffusion term $D_{\mathrm{pCMFD}}=\frac{1}{3 \Sigma_{t r}}+\frac{\Delta}{4}$, where $\Delta$ denotes the coarse mesh size. Although the pCMFD method is unconditionally stable for monoenergetic infinite homogeneous problems, it may become less efficient than CMFD for problems with intermediate or smaller coarse mesh sizes[7]. The odCMFD was proposed and implemented in the MPACT code[8] by Zhu[3], which can be regarded as a generalization of pCMFD by adding an artificial term $\theta \Delta$ rather than a fixed $\Delta / 4$ as in pCMFD to the diffusion coefficient, where $\theta$ denotes an adaptive number. The Fourier analysis and numerical results based on a monoenergetic infinite homogeneous medium problem showed that the optimal $\theta$ increases with the optical thickness increasing and is always smaller than the value $1 / 4$ predicted by pCMFD.

Recently, a category of CMFD methods, regarded as "higher-order" prolongation methods (hpCMFD) [9], was proposed to use both the local and neighboring coarse mesh fluxes to update the fine cell flux. One of the hpCMFD methods, refered to lpCMFD, was proposed by Wang and Xiao[4] to use a linear prolongation to update the fine cell scalar fluxes, which will be discussed in the next section. It showed that lpCMFD is more stable than conventional CMFD methods and is effective for a wide range of optical thicknesses. A Fourier analysis of a $S_{N^{-}}$CMFD scheme with linear prolongation performed by Shen[9] showed that lpCMFD is unconditionally stable for fixed source problems and k-eigenvalue problems. A variant method based on the fine cell optical thickness threshold and insights provided by odCMFD was developed to improve the stability of lpCMFD.

The Method of Characteristics(MOC) is a very popular method to solve neutron transport equations. Li[5] demonstrated a similar linear prolongation technique in openMOC [10] by simply performing a linear interpolation using the prolongation factors of neighboring cells, which is limited to sector-shaped fine mesh cells. In this paper, we implemented lpCMFD into the MOC code MPACT for a variety of fine meshes and the results are summarized in this paper. A track-based centroids calculation method is introduced to find the centroids coordinates for random shapes of fine cells. And the numerical results are provided to demonstrate the stability and efficiency of lpCMFD method on MOC.

\section{IpCMFD on MOC}

Considering the multi-group steady state Boltzmann neutron transport equation:

$$
\begin{aligned}
\vec{\Omega} \cdot \vec{\nabla} \psi(\vec{x}, \vec{\Omega})+\Sigma_{t, g}(\vec{x}) \psi_{g}(\vec{x}, \vec{\Omega}) & = \\
& \sum_{g^{\prime}=1}^{G} \int_{0}^{4 \pi} \Sigma_{s, g^{\prime} \rightarrow g}\left(\vec{x}, \vec{\Omega} \cdot \vec{\Omega}^{\prime}\right) \psi_{g^{\prime}}\left(\vec{x}, \vec{\Omega}^{\prime}\right) \mathrm{d} \Omega^{\prime}+\frac{1}{4 \pi} \frac{\chi_{g}(\vec{x})}{k_{\text {eff }}} \sum_{g^{\prime}=1}^{G} \nu \Sigma_{f, g^{\prime}}(\vec{x}) \phi_{g^{\prime}}(\vec{x})
\end{aligned}
$$

where $\psi$ represents the angular flux while $\phi$ represents the scalar flux. The discrete ordinates approximation is used for the angular variables, and the equation are solved for the chosen set of directions. The flux distribution is then obtained based on the solution on the characteristic rays. This is the essence of Method of Characteristic (MOC). 
The problem domain is divided into coarse cells and the coarse cells are homoginized. The angularintegrated neutron balance equation can then be written as:

$$
\nabla \cdot\left(\frac{-1}{3 \Sigma_{t, C M} \nabla+\hat{D}_{C M}^{l+1 / 2}}\right) \phi^{l+1}+\left(\Sigma_{t, C M}-\Sigma_{s, C M}\right) \phi^{l+1}=Q
$$

where $\phi^{l+1}$ is the coarse mesh flux at the $l^{\text {th }}$ MOC iteration, $\Sigma_{t, C M}$ and $\Sigma_{s, C M}$ are the total and scattering cross section of the coarse mesh. $\hat{D}_{C M}^{l+1 / 2}$ is the drift coefficent which can be obtained by

$$
\hat{D}_{C M}^{l+1 / 2}=\frac{\int \mathrm{d} \Omega \vec{\Omega} \psi^{l+1 / 2}+\frac{1}{3 \Sigma_{t, C M}} \nabla \bar{\phi}^{l+1 / 2}}{\phi^{l+1 / 2}}
$$

where $\bar{\phi}^{l+1 / 2}$ is the coarse cell-averaged scalar flux at the $l^{\text {th }}$ MOC iteration. Eq.(2) can be solved to obtain the flux of the coarse cells. The coarse mesh flux can then be used to update the scalar flux in Eq.(1) so that MOC iteration can be effectively accelerated.

Conventionally, the scalar flux $\phi$ in Eq. 1 is updated directly by

$$
\phi^{l+1}=\phi^{l+1 / 2} \frac{\Phi^{l+1}}{\bar{\phi}^{l+1 / 2}}
$$

In this paper, a linear prolongation is applied to update $\phi$ :

$$
\phi^{l+1}=\phi^{l+1 / 2}+\delta \phi
$$

where $\delta \phi$ is defined as

$$
\delta \phi=\Phi^{l+1}-\bar{\phi}^{l+1 / 2}
$$

and can be obtained by linear interpolation using the delta fluxes of the neighbor cells as depicted in Fig. 1(a).

In 2D case, to find the delta flux $\delta \phi_{x y}$ at point $(x, y)$ in coarse cell $(i, j)$, the delta fluxes at the four corners of coarse cell $(i, j)$ is obtained first. And the delta flux at each corner is the averaged delta flux of the four coarse cells which share the same vertex. For example,

$$
\delta \phi_{s w}=\frac{1}{4}\left(\delta \phi_{i-1, j}+\delta \phi_{i, j}+\delta \phi_{i-1, j+1}+\delta \phi_{i, j+1}\right)
$$

However, for the case in which the coarse cell is at the boundary, some delta flux values in the right-hand-side of Eq. (7) may be missed. To handle with this issue, a layer of ghost coarse cells is introduced to the original coarse mesh. And the value of delta flux of a ghost cell depends on the corresponding boundary condition:

$$
\delta \phi_{\text {ghost }}=\alpha \delta \phi_{\text {boundary cell }}
$$

where $\alpha$ is the albedo of the boundary. For reflective boundary condition, $\alpha=1$; for vacuum boundary condition, $\alpha=0$.

Thus $\delta \phi_{x y}$ is obtained by bi-linear interpolation of $\delta \phi_{n w}, \delta \phi_{n e}, \delta \phi_{s w}$ and $\delta \phi_{s e}$ :

$$
\delta \phi_{x y}=a x+b x y+c y+d
$$




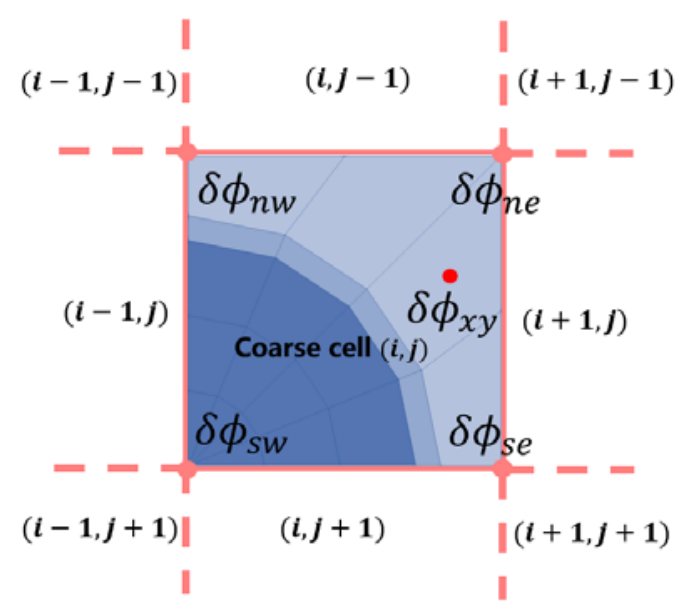

(a) Linear interpolation to get $\delta \phi$.

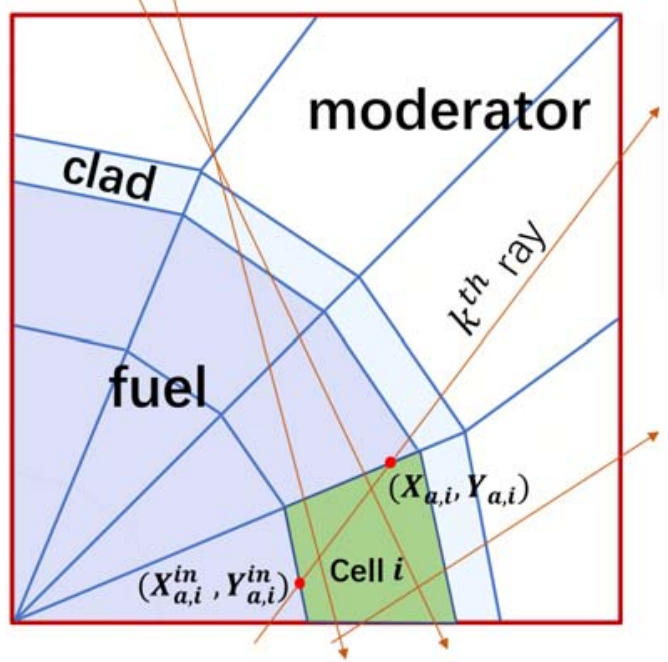

(b) Ray-tracing in a coarse cell.

Figure 1: lpCMFD $\delta \phi$ interpolation and track-based centroids calculation method.

where

$$
\begin{aligned}
a & =\frac{\delta \phi_{s e}-\delta \phi_{s w}}{\Delta x} \\
b & =\frac{\delta \phi_{n e}-\delta \phi_{n w}+\delta \phi_{s w}-\delta \phi_{s w}}{\Delta x \Delta y} \\
c & =\frac{\delta \phi_{n w}-\delta \phi_{s w}}{\Delta y} \\
d & =\delta \phi_{C}
\end{aligned}
$$

\section{Track-based Centroid Calculation}

In the MOC method, the centroids coordinates of a region can be calculated with a track-based method[11]. Fig. 1(b) illustrates the ray tracing process in a quarter pin cell, which is regarded as a coarse cell in CMFD. The fuel region is divided into eight regions and the cladding region and moderator region are divided into four regions respectively. Note that the rays in Fig. 1(b) only represents a part of characteristic rays for four angles. In the actual MOC sweeper, there are tens to hundreds of parallel rays for each angle that traverses the whole cell.

Assuming the fine cell we are interested in is the green cell. A characteristic ray with an azimuthal angle $a$ enters the cell $i$ from the point $\left(X_{a, i}^{i n}, Y_{a, i}^{i n}\right)$, and goes out from the point $\left(X_{a, i}, Y_{a, i}\right)$. In an MOC sweeper, the coordinates of the outgoing point $\left(X_{a, i}, Y_{a, i}\right)$ are usually unknown. But it can be determined with the incoming point coordinates $\left(X_{a, i}^{i n}, Y_{a, i}^{i n}\right)$ and track length $s_{m, i, k}$, as described by Eq. (11).

$$
X_{a, i}=\frac{s_{m, i, k} \cos \varphi_{a}}{\sin \theta_{p}}+X_{a, i}^{i n}, \quad Y_{a, i}=\frac{s_{m, i, k} \sin \varphi_{a}}{\sin \theta_{p}}+Y_{a, i}^{i n}
$$


where $\varphi_{a}$ is the azimuthal angle and $\theta_{p}$ is the polar angle of the ray. The subscript $m=(p, a)$ represents the angle of the ray, including polar angle $p$ and azimuthal angle $a ; i$ represents the cell number and $k$ represents the ray number.

In practical application, the scaled track length $t_{m, i, k}$ instead of $s_{m, i, k}$ is usually used. And the relation between $t_{m, i, k}$ and $t_{m, i, k}$ is given as:

$$
t_{m, i, k}=\xi_{a, i} s_{m, i, k}
$$

where $\xi_{a, i}$ is the renormalization factor and in 2D case, $\xi_{a, i}$ is defined as the ratio of the cell's actual area to the area that all rays in azimuthal angle $a$ traversed:

$$
\xi_{a, i}=\frac{S_{i}}{\delta d_{a} \sum_{k} s_{a, i, k}}
$$

where $S_{i}$ is the area of cell $i ; \delta d_{a}$ is the distance between the rays with azimuthal angle $a$ and $s_{a, i, k}$ is the track length of the $k$-th ray with azimuthal angle $a$ in cell $i$.

In the MOC method, an integration over cell $i$ can be numerically evaluated using track data, as shown by Eq. (14)

$$
\begin{aligned}
\langle f, g\rangle_{m, i} & =\frac{\sin \theta_{p}}{S_{i}} \sum_{k} \int_{0}^{t_{m, i, k}} f\left(t_{m}^{\prime}\right) g\left(t_{m}^{\prime}\right) \mathrm{d} t_{m}^{\prime} \delta d_{a} \\
\langle f, g\rangle_{i} & =4 \pi \sum_{m} \omega_{m}\langle f, g\rangle_{m, i}
\end{aligned}
$$

where $\omega_{m}$ is the quadrature weight in direction $m$ corresponding to the product quadrature set chosen in the MOC sweeper.

Thus, the angular-dependent coordinates of the centroid of cell $i\left(X_{a, i}^{C}, Y_{a, i}^{C}\right)$ can be calculated by Eq. (15), which can not only be applied to calculate the centroid of a polygon cell as depicted in Fig. 1(b), but can also be applied for any other shapes of cells.

$$
X_{a, i}^{C}=\left\langle 1, X_{a, i}\right\rangle_{a, i}, \quad Y_{a, i}^{C}=\left\langle 1, Y_{a, i}\right\rangle_{a, i}
$$

\section{IpCMFD Algorithm in MOC}

Compared with the conventional CMFD, it is necessary in lpCMFD to determine the neighboring cells for all the coarse cells and calculate the centroids coordinates for all the fine cells at the beginning. Another difference lies in the step where fine cell fluxes are updated with flux results in coarse cells. For lpCMFD, the fluxes in fine cells are updated with Eq. (5) while for conventional CMFD, they are updated with Eq. (4).

Note that the first two steps are only needed to be performed once prior to the transport iterations. The results are stored and then applied in the MOC iterations when needed. Compared to the conventional CMFD, the flux updating step in lpCMFD only requires one more linear interpolation calculation, as formulated in Eq. (9) and Eq. (10), which can also be quickly done. Therefore, lpCMFD will not increase the total calculation cost significantly. 


\section{Numerical Results}

The 2D C5G7 benchmark is a well established problem that is widely used for the verification of neutron transport code[12]. In this section, a 2D C5G7 problem is performed to verify lpCMFD method on MOC. The results of conventional CMFD and odCMFD are provided as well for comparison. The model of the 2D C5G7 problem is 1/4 of a whole core, as depicted by Fig. 2.

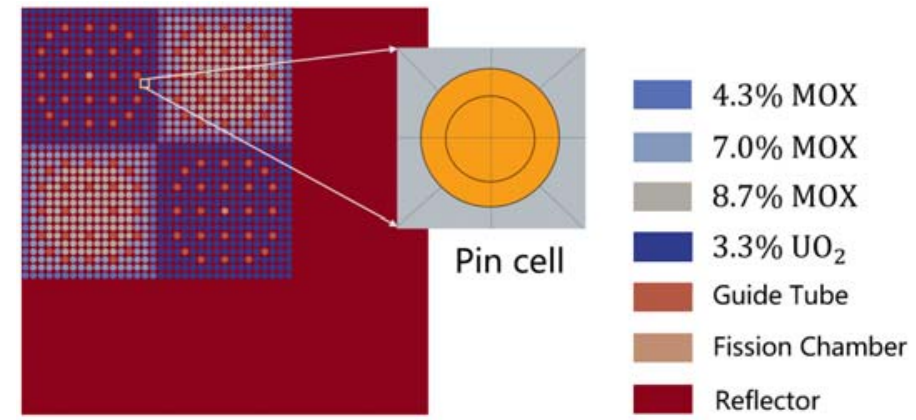

Figure 2: Geometry and fuel configuration of the 2D C5G7 problem.

The 2D C5G7 benchmark is a heterogeneous problem consisting of $\mathrm{UO}_{2}$ and mixed oxide(MOX) fuel. The 1/4 C5G7 core contains 9 assemblies, which are two $\mathrm{UO}_{2}$ assemblies, two MOX fuel assemblies and five reflector assemblies. Each fuel assembly contains $17 \times 17$ fuel pins and the configuration of fuel pins is shown in Fig. 2. The size of each pin cell is $1.26 \mathrm{~cm} \times 1.26 \mathrm{~cm}$ and the radius of the fuel pin with cladding is $0.54 \mathrm{~cm}$. Each pin cell is divided into 16 fine cells as illustrated by the pin cell mesh in the upper right corner of Fig. 2. The fuel region is divided into two concentric rings with four fine cells in each ring while the moderator region is divided into eight fine cells.

For the whole 1/4 core, the upper and left boundaries are set to reflective boundary condition while the other two boundaries are set to vacuum boundary condition due to the symmetry in the core.

Table 1: $k_{\text {eff }}$ and iteration cycles for different CMFD schemes, 2D C5G7 problem

\begin{tabular}{cccccc}
\hline Scheme & iterations & $k_{\text {eff }}$ & 2-norm residual & max power & max power relative error \\
\hline without CMFD & 1257 & 1.187162 & $9.970158 \mathrm{E}-11$ & 2.490228 & (used as ref.) \\
CMFD & - & Diverge & - & - & - \\
odCMFD & 37 & 1.1871162 & $7.753420 \mathrm{E}-11$ & 2.490228 & $6.73 \mathrm{E}-8$ \\
lpCMFD diff interp & 26 & 1.1871142 & $8.323320 \mathrm{E}-11$ & 2.490244 & $6.53 \mathrm{E}-6$ \\
lpCMFD ratio interp & 24 & 1.1871141 & $6.515899 \mathrm{E}-11$ & 2.490245 & $6.92 \mathrm{E}-6$ \\
\hline
\end{tabular}

The number of MOC outer iterations for different CMFD schemes are listed in Table. 1. The last column "max power relative error" are compared with the original results without CMFD. And 


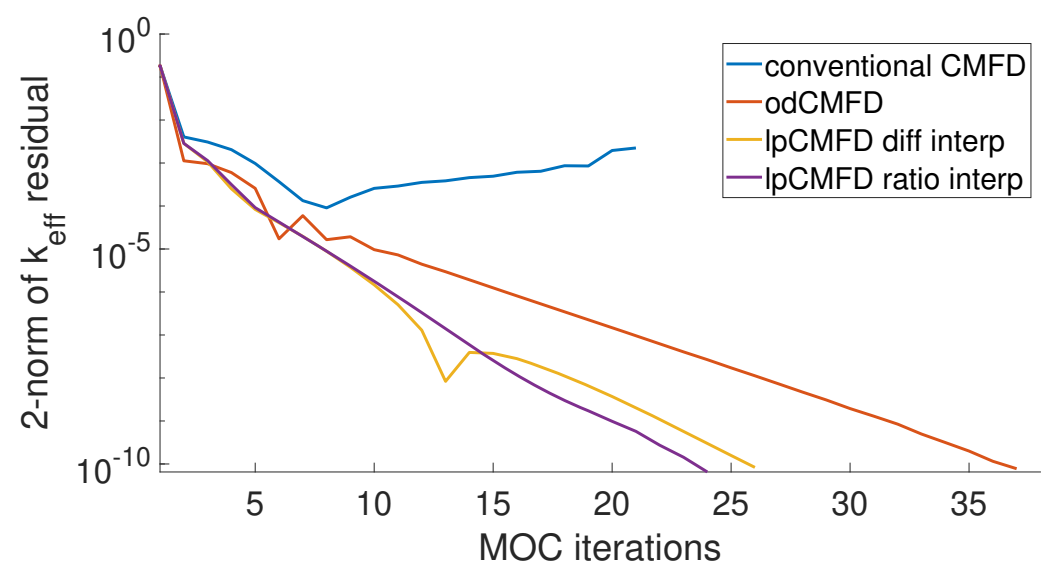

Figure 3: 2-norm of $k_{\text {eff }}$ residuals vs. MOC iterations, 2D C5G7 problem.

the last row "lpCMFD ratio interp" refers to using linear interpolation on the ratio of fluxes $\frac{\Phi^{l+1}}{\phi^{l+1 / 2}}$ instead of the difference $\delta \phi=\Phi^{l+1}-\bar{\phi}^{l+1 / 2}$. Fig. 3 shows how the residuals of $k_{\text {eff }}$ decreases as the MOC iteration number increases. As indicated, the 2D C5G7 problem is unstable for conventional CMFD and fails to converge. odCMFD and lpCMFD can both stabilize the CMFD algorithm and achieve converged results, while lpCMFD performs better than odCMFD. The linear interpolation for the ratio of fluxes is also applied and the result shows it can reduce two more iterations compared to using linear interpolation for the difference of fluxes for this problem. However, the ratio interpolation method would become unstable when the number of fine meshes in a coarse cell becomes small, compared to the difference based interpolation method.
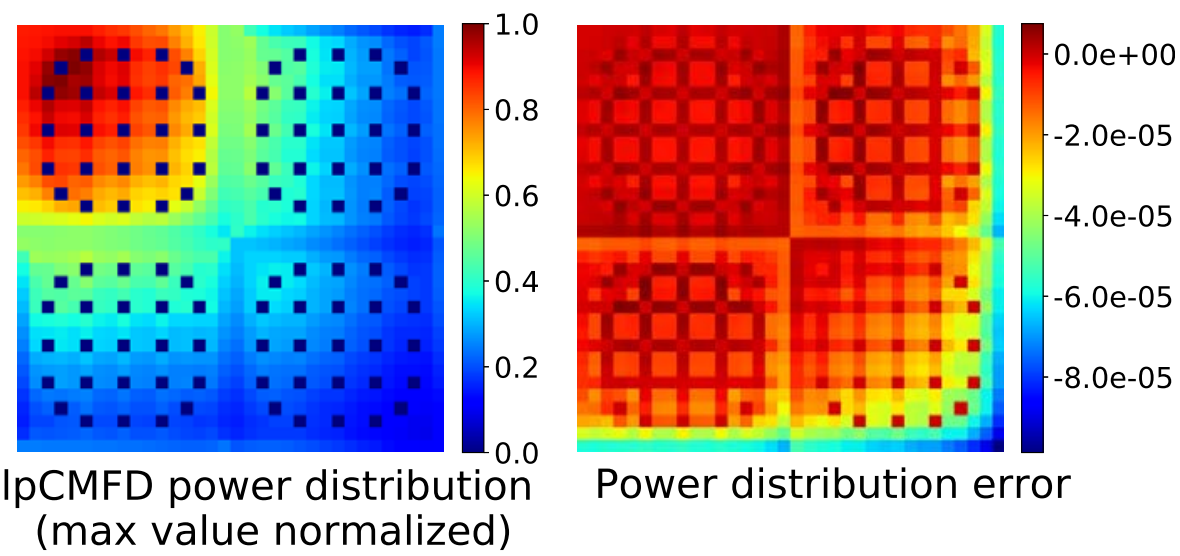

Power distribution error

Figure 4: Power distribution and relative errors of the 2D C5G7 problem.

The maximum value normalized power distribution of the 2D C5G7 problem and the relative error(odCMFD results as reference) are illustrated in Fig. 4. The result shows that lpCMFD obtains nearly the same power distribution as odCMFD. With regard to the run time, lpCMFD takes nearly the same time per iteration as odCMFD and there is no remarkable difference (less 
than $1 \%)$.

\section{Conclusion}

The linear prolongation CMFD method is applied on a MOC code. A track-based method is applied to calculate the centroids coordinates for the fine cells. The numerical results of the 2D C5G7 problem demonstrate that lpCMFD can stabilize the CMFD iterations on MOC method effectively and lpCMFD method performs better than odCMFD on reducing the outer MOC iterations.

\section{REFERENCES}

[1] K. Smith. "Nodal method storage reduction by nonlinear iteration." volume 44 (1983).

[2] N. Z. Cho, G. S. Lee, and C. J. Park. "On a new acceleration method for 3D whole-core transport calculations." Genshikaku Kenkyu, volume 48(2), pp. 79-80 (2003). URL http: //inis.iaea.org/Search/search.aspx?orig_q=RN:35014836.

[3] A. Zhu, M. Jarrett, Y. Xu, B. Kochunas, E. Larsen, and T. Downar. "An optimally diffusive Coarse Mesh Finite Difference method to accelerate neutron transport calculations." Annals of Nuclear Energy, volume 95, pp. 116-124 (2016). URL http://www.sciencedirect.com/ science/article/pii/S030645491630250X.

[4] D. Wang and S. Xiao. "A Linear Prolongation Approach to Stabilizing CMFD." Nuclear Science and Engineering, volume 190(1), pp. 45-55 (2018). URL https://doi.org/10.1080/ 00295639.2017.1417347.

[5] L. Li, K. Smith, and B. Forget. "Techniques for Stabilizing Coarse-Mesh Finite Difference (CMFD) in Methods of Characteristics (MOC)." Prof Forget via Chris Sherratt (2015). URL http://dspace.mit.edu/handle/1721.1/109272.

[6] Y. Jung and H. Joo. "Decoupled planar MOC soluition for dynamic group constant generation in direct three-dimensional core calculations." volume 4, pp. 2157-2167 (2009).

[7] N. Z. Cho. "The partial current based CMFD (p-CMFD) method revisited." In Proceedings of the KNS autumn meeting, pp. 1CD-ROM. KNS, Korea, Republic of (2012). URL http: //inis.iaea.org/Search/search.aspx?orig_q=RN:44062577.

[8] B. Kochunas, B. Collins, D. Jabaay, T. Downar, and W. Martin. "Overview of development and design of MPACT: Michigan parallel characteristics transport code." volume 1, pp. 4253. American Nuclear Society - ANS, United States (2013).

[9] Q. Shen, Y. Xu, and T. Downar. "Stability analysis of the CMFD scheme with linear prolongation." Annals of Nuclear Energy, volume 129, pp. 298-307 (2019). URL http://www.sciencedirect.com/science/article/pii/S0306454919300805.

[10] W. Boyd, S. Shaner, L. Li, B. Forget, and K. Smith. "The OpenMOC method of characteristics neutral particle transport code." Annals of Nuclear Energy, volume 68, pp. 43-52 (2014). URL http://www.sciencedirect.com/science/article/pii/S0306454913006634.

[11] R. M. Ferrer and J. D. Rhodes. "The linear source approximation and particle conservation in the Method of Characteristics for isotropic and anisotropic sources." Annals of Nuclear Energy, volume 115, pp. 209-219 (2018). URL http://www.sciencedirect.com/science/article/ pii/S0306454918300203.

[12] E. Lewis, M. Smith, and N. Tsoulfanidis. "Benchmark specification for deterministic 2-D/3D MOX fuel assembly transport calculations without spatial homogenisation (C5G7 MOX)." OECD/NEA Report (2001). 